Revista de Psicología Vol. 31 (2), 2013 (ISSN 0254-9247)

\title{
Validación de una escala de medición del consenso estratégico en equipos pedagógicos
}

\author{
Katia Puente-Palacios ${ }^{11}$ y Tamara Puente ${ }^{22}$ \\ Universidad de Brasilia
}

Se estudia las evidencias psicométricas de validez de una escala de evaluación del consenso estratégico en equipos pedagógicos. El consenso estratégico es un proceso grupal descrito como la concordancia entre los miembros sobre asuntos estratégicos para el éxito del trabajo. Los participantes fueron 140 profesores de escuelas de Quito (Ecuador). La escala aplicada cuenta con 14 ítems y los análisis estadísticos realizados revelaron una estructura unifactorial que explica $49.72 \%$ de la variancia. La confiabilidad interna fue satisfactoria $(\alpha=0.92$; $r_{\text {item-total }}=0.66$ ). Los resultados revelan que la escala constituye una herramienta útil para el diagnóstico del consenso existente entre miembros de equipos pedagógicos y contribuye para la mejor comprensión del fenómeno.

Palabras clave: consenso estratégico, equipos pedagógicos, validación psicométrica, análisis factorial.

\section{Development of a measurement scale of strategic consensus on pedagogic teams}

Strategic consensus is a group process that can be described as the agreement among team members on strategic matters for success at work. The psychometric evidences of the validity of an assessment scale measuring strategic consensus in pedagogic teams are analyzed. Participants were 140 school teachers in Quito (Ecuador). Statistical analyses of the applied scale containing 14 items revealed a uni-dimensional factor structure explaining $49.7 \%$ of the variance. The internal consistency of the scale was satisfactory $(\alpha=.92$; $r_{\text {item-total }}=.66$ ). Results show that the scale is an useful tool for the diagnosis of the existence of consent among members of pedagogic teams and contributes to a full understanding of the phenomenon.

Keywords: strategic consensus, pedagogic teams, psychometric validation, factor analysis.

$1 \quad$ PhD en Psicología por la Universidad de Brasilia (Brasil) y catedrática de posgrado (Maestría y Doctorado) en Psicología Social, del Trabajo y de las Organizaciones de la Universidad de Brasilia. Contacto: Universidad de Brasilia, Instituto de Psicología, Campus Darcy Ribeiro. Brasilia-DF. CEP-70910-900; kep.palacios@gmail.com

$2 \mathrm{MsC}$ en Ciencias de la Educación por la Pontificia Universidad Católica del Ecuador y docente en la Facultad de Ciencias de la Educación. Contacto: tamarapuen@yahoo.es 

En el escenario de las organizaciones es cada vez más frecuente la presencia de equipos de trabajo. Puente-Palacios y González-Romá (2013) relatan que $90 \%$ de las empresas americanas consideradas grandes poseen en su estructura organizativa equipos de trabajo. A pesar de que en el Ecuador no se tenga datos sistemáticos sobre esa práctica, la observación de contexto laboral revela también la presencia de equipos. En un estudio descriptivo realizado por Arteaga y Lasio (2009) sobre los factores asociados al dinamismo empresarial en el Ecuador, destacan que el trabajo en equipos es una de las prácticas reportadas por los empresarios; por tanto, las evidencias locales también revelan adopción de equipos de trabajo como unidades de desempeño. En el ámbito educativo la situación no es diferente: la meta de trabajo establecida es compleja y multifacética, lo que torna necesaria la convergencia de esfuerzos de un conjunto de personas para que su alcance sea viable. Así, se puede afirmar que los equipos constituyen unidades ampliamente utilizadas en los más diversos tipos de organizaciones. En esos equipos de trabajo, son diversos los procesos protagonizados por sus integrantes: en el transcurso del ciclo de su desarrollo puede ser observado el surgimiento de actitudes colectivas, patrones de comunicación propios del equipo e inclusive formas similares de percibir e interpretar el entorno laboral. Esa semejanza es referida por la literatura del área como consenso que puede desembocar en ventajas o perjudicar al equipo, y para investigar empíricamente el papel del consenso estratégico, el primer paso es poseer un instrumento de medida que al ser adecuado y fiable pueda ofrecer datos confiables sobre ese fenómeno y sobre los efectos que ejerce sobre otros atributos del equipo de trabajo.

El objetivo del estudio realizado fue adaptar una escala de medida de consenso sobre las prácticas pedagógicas, investigando los índices de confiabilidad de la escala en una muestra de docentes de centros educativos del Municipio de Quito. Los equipos de trabajo 
se conceptualizaron como entidades sociales completas, insertas en un contexto organizativo mayor, compuestas de por lo menos tres personas a quienes son atribuidos diferentes papeles, pero que actúan de manera interdependiente, revelando relaciones personales y de trabajo dinámico y complejo. Teniendo la comprensión de esa estructura como característica de los equipos, investigadores de este campo de conocimiento afirman que el éxito del trabajo del equipo deriva de varios elementos entre los que destacan: (a) las características de los miembros, (b) el grupo como un todo, (c) el ambiente en que operan y también (d) las interacciones y relaciones que a cada momento son construidas en el equipo (Hackman, 1987).

Entre los factores individuales se encuentran personalidad, habilidades y actitudes; entre los grupales se mencionan aspectos como la diversidad demográfica y el tamaño del equipo; finalmente, entre los ambientales consta la estructura de recompensas adoptada por la organización, las oportunidades de participación en actividades de entrenamiento y en general, las políticas de la organización. Al mencionar las relaciones e interacciones protagonizadas por los miembros se enfatiza el papel de la comunicación, el soporte social ofrecido, la forma de administrar el conflicto y la definición de estrategias de trabajo, entre otras. En las relaciones de interacción protagonizadas en los equipos, debe ser enfatizado que ciertos procesos vivenciados por los miembros son dinámicos y sinérgicos, de tal forma que después de alcanzado el estado de desarrollo no se trata más de la contribución que una u otra persona ofrece al trabajo grupal. Se trata de aquello que el grupo construye colectivamente. Esas manifestaciones grupales pasaron a ser denominadas como estados emergentes (Mathieu, Maynard, Rapp \& Gilson, 2008) y entre ellos pueden ser encontradas las llamadas cogniciones compartidas. Swaab, Postmes, Van Beest y Spears (2007) describen las cogniciones compartidas como estructuras de conocimiento común o congruente, que pueden referirse a diversos aspectos, tales como la tarea del grupo, las estrategias de trabajo o incluso, las relaciones establecidas con el medio interno y externo a la organización. 
Un tipo específico de cognición compartida es denominado como consenso estratégico y considerando la importancia de ese fenómeno, cada vez pueden ser encontrados con más frecuencia reportes científicos que lo toman como objeto de estudio. Kellermanns, Eddleston, Barnett y Pearson (2008) lo definen como el grado de acuerdo o concordancia que existe en un equipo en relación a las estrategias adoptadas por los miembros para desempeńar las tareas y alcanzar la meta de trabajo. Los mismos autores defienden que a pesar de ser un tema reciente en estudios empíricos, el consenso estratégico está siendo considerado de extrema importancia. Esto porque al existir un alto nivel de concordancia o acuerdo en el equipo, aumentan también las probabilidades de que surja mayor cooperación entre los miembros. Como consecuencia habría reducción del tiempo que el grupo gasta para llegar a tomar una decisión y, de esa forma, se favorecería el buen desempeño del equipo. Aun con esas consideraciones, es importante destacar que el consenso estratégico no es siempre un factor deseable, pues no son solo positivas sus consecuencias. En ciertos casos, por ejemplo, el consenso constante en el equipo puede inducir a los miembros a optar por respuestas comunes o usuales que están acostumbrados a ofrecer. Puede también ser antecedente de decisiones pobres y poco creativas. Las consideraciones hechas permiten manifestar que la ausencia de consenso y la presencia de discordancia, así como la existencia de puntos de vista diferenciados pueden promover la discusión, que si es adecuadamente administrada y organizada, podrá a resultar en respuestas más innovadoras y creativas.

Como resultado de las afirmaciones realizadas, se concluye que el consenso no es positivo ni negativo en sí mismo. Depende tanto del contenido como del escenario de trabajo. Por ejemplo, concordar sobre la importancia de la meta y de las estrategias de trabajo es fundamental pues si los miembros no consideran la meta relevante o si no concuerdan con la forma de trabajar, no pueden ser esperados resultados positivos. Por otro lado, discordar con las decisiones y buscar nuevas respuestas a los problemas de trabajo, presentando puntos de vista que antes no fueron considerados, puede ser extremamente positivo si el equipo enfrenta desafíos inusuales o si lo que se busca es una respuesta creativa. 
Por tanto, al discutir las consecuencias del consenso es imprescindible destacar el contenido del consenso. Revisando los estudios empíricos publicados sobre el tema, observamos algunos resultados interesantes. Kellermanns et al. (2008) relatan que los principales elementos explicativos del consenso que ya se han estudiado son las características demográficas de los integrantes del grupo, la naturaleza del proceso de decisión, y la estructura organizativa. Según esos autores, los estudios de campo han revelado que hay relación positiva entre la semejanza de atributos demográficos (sexo, edad) y el consenso estratégico. Eso significa que cuanto más parecidos los miembros del equipo, mayor la probabilidad de que entren en acuerdo. En otras investigaciones se ha estudiado las relaciones entre consenso y sus resultados. De esa manera, estudios de base empírica han identificado que la existencia de consenso ayuda al aumento de la cooperación y cohesión del grupo, así como la habilidad de obtener consenso en ocasiones futuras.

Sin embargo, por tratarse de un fenómeno que normalmente es investigado en equipos de alto nivel en la organización, como los equipos de gerencia, la mayor parte de los estudios buscó establecer relaciones entre el consenso de esos gerentes y el desempeńo global de la organización. Como consecuencia, esos resultados no pueden generalizarse a niveles más bajos de la empresa, como los mandos medios o los equipos operativos. Otro problema observado es el hecho de que, en buena parte de los estudios, la variable desempeño fue medida de maneras diferentes, lo que dificulta la comparación genuina de los resultados (Kellermanns et al., 2008). La revisión de estudios empíricos también muestra que no existe una tendencia teórica única al estudiar el consenso estratégico. Algunos autores buscan entender la manera en que se forma el consenso, esto es, desean responder a preguntas relacionadas al origen del consenso en el proceso de tomar decisiones grupales. Por otro lado, autores también buscan obtener respuestas sobre cuáles son los impactos que pueden ser esperados de la presencia de consenso en el desempeño organizativo. En todos los casos, para dar inicio a los estudios es imprescindible contar con la herramienta que permita efectuar la medición de la intensidad del consenso. Así, tan importante como 
estudiar los antecedentes o las consecuencias del consenso, es estudiar las propiedades de las escalas propuestas para medirlo.

Específicamente, desde una perspectiva teórica, en este trabajo adoptamos las prácticas pedagógicas como contenido central del consenso a ser investigado. Ese consenso es comprendido como el acuerdo existente entre los miembros del equipo pedagógico sobre las prácticas de trabajo establecidas buscando alcanzar las metas de desempeño en la institución escolar. Sobre esas prácticas es importante destacar que ellas son comprendidas como las estrategias de actuación del equipo a partir de las cuales se espera realizar efectivamente la meta de trabajo. La lógica subyacente establece que en la medida en que los miembros del equipo concuerdan con los planes de acción (prácticas pedagógicas) adoptadas por la escuela para incentivar el desarrollo integral de los estudiantes, mayor probabilidad existe de que los resultados del desempeño del equipo sean de hecho alcanzados. Así, cuanto mayor concordancia con las estrategias de trabajo, mejores resultados deben ser esperados. Sin embargo, para verificar la adecuación de esa expectativa teórica a la realidad, es imprescindible la realización de un estudio empírico, de diseño relacional. Para tanto, el primer paso es contar con las herramientas de medida adecuadas al contexto donde el estudio será realizado. Al cabo de una exhaustiva verificación realizada en las bases de datos Scielo, Redalic y Pepsic que contienen publicaciones científicas de América Latina, se constató la ausencia de instrumentos locales en español, con evidencias de validez psicométrica, que permitan diagnosticar con un margen de seguridad adecuado, la existencia de consenso en equipos pedagógicos, específicamente en relación a las prácticas adoptadas por la escuela. Más aún, considerando que el proceso de traducción y adaptación de un instrumento de medida psicológica debe considerar la realidad del escenario local (Cassep-Borges, Balbinotti \& Teodoro, 2010), se observa la necesidad de un estudio empírico que considere la adaptación e investigación de las evidencias de confiabilidad y precisión de la escala traducida, por cuanto las herramientas de medición de atributos psicológicos — como comportamientos, actitudes y afectos-, pueden ser influenciados por la cultura local. 


\section{Método}

\section{Participantes}

Los 140 participantes del estudio (70.7\% varones y $28.2 \%$ mujeres) se seleccionaron a partir de los cuestionarios entregados a 183 profesores de 9 escuelas particulares, laicas y religiosas localizadas en la ciudad de Quito, con una tasa de respuesta útil de $76.50 \%$. Los 43 sujetos descartados devolvieron el cuestionario en blanco, o con más del 50\% de preguntas sin respuesta, o señalaron la misma respuesta a todas las preguntas (invariancia absoluta). El 43.6\% de los participantes contaban con nivel educativo de nivel superior completo, especialmente en áreas pedagógicas, seguido por superior completo en otras áreas $(27.9 \%)$ y pertenecían mayoritariamente a escuelas particulares religiosas $(57.1 \%)$, según se observa en la Tabla 1.

\section{Tabla 1}

Porcentajes de atributos de los miembros de la muestra

\begin{tabular}{lcc}
\hline Atributos & Cantidad & Porcentaje \\
\hline Sexo & 99 & 70.7 \\
Masculino & 40 & 28.6 \\
Femenino & & \\
\hline Tipo de escuela & 60 & 42.9 \\
$\quad$ Particular laica & 80 & 57.1 \\
Particular religiosa & & \\
Nivel de educación & 4 & 2.9 \\
Secundaria completa & 6 & 4.3 \\
Superior incompleto & 39 & 27.9 \\
Superior completo & 13 & 9.3 \\
Superior incompleto en áreas pedagógicas & 61 & 43.6 \\
Superior completo en áreas pedagógicas & 17 & 12.1 \\
Posgrado & & \\
\hline
\end{tabular}


Los datos presentados también revelan que los participantes del estudio tienden a permanecer en el mismo empleo (tiempo medio de siete ańos) y trabajan en equipos que pueden ser considerados de tamaño medio (36 personas), a pesar de que se observa una considerable variabilidad en ese aspecto, pues hubo sujetos que afirmaron trabajar en equipos compuestos por dos personas, mientras otros afirmaran pertenecer a equipos compuestos por 80 miembros. El aspecto favorable de los datos comentados es la antigüedad, pues la existencia de consenso - fenómeno a ser investigado por la escala—, solo puede ser buscada en equipos de trabajo en los que haya un considerable tiempo de convivencia que permita la construcción de interpretaciones similares entre los miembros del equipo sobre el escenario de trabajo en que laboran, lo que es imprescindible para el estudio propuesto.

\section{Medidas e instrumentos de medida}

El consenso estratégico en equipos pedagógicos fue medido a partir de la escala de evaluación del consenso estratégico desarrollado por Moreira (2011), compuesta por 14 afirmaciones que fueron traducidas del portugués al español utilizando el método de doble traducción. Posteriormente, las versiones en portugués fueron comparadas con el fin de evaluar las diferencias entre la redacción original y la traducida doblemente. Luego, se realizó el procedimiento de análisis de adecuación semántica; para ello, fueron entrevistados seis docentes con perfil similar al que sería esperado de los profesores miembros de equipos pedagógicos a los cuales se destina el instrumento. Las dudas que surgieron en esa fase y que fueron mencionadas por los entrevistados, dieron origen a la realización de ajustes en la redacción de los ítems hasta que fuera considerado que el enunciado era satisfactorio. De esa forma, la escala aplicada estuvo compuesta por 14 ítems, escritos en la forma de afirmaciones que deberían ser respondidas en una escala tipo Likert de concordancia de 5 puntos, en la cual el valor 1 equivale a responder nada (la afirmación no refleja las prácticas adoptadas por la escuela) y 5 equivale a concordancia total (la afirmación refleja totalmente la forma 
de actuación de la escuela). El cuestionario también contenía seis preguntas sobre aspectos demográficos y funcionales que permitieron la caracterización de la muestra.

\section{Procedimiento}

Primero, los miembros del equipo de investigación visitaron algunos establecimientos educacionales — cuyos nombres serán mantenidos en sigilo respetando el derecho al anonimato-, con el fin de entrar en contacto con los docentes participantes. Se les explicó el objetivo y la naturaleza de la participación y se realizó la demostración de llenado de los cuestionarios. Se siguieron los estándares éticos para la investigación con seres humanos, destacándose la voluntariedad y el anonimato de la participación evitando colocar señas que pudieran identificar al participante. Asimismo, se enfatizó que la recolección de datos era grupal, sin acceso por parte de la escuela a las respuestas individuales.

Los cuestionarios fueron entregados por miembros del equipo de investigación y posteriormente ellos los recogieron directamente de los participantes o de otra persona que se encargó de recogerlos, manteniéndolos en sobre cerrado y sin identificación. Los datos recogidos fueron tabulados en el programa Excel y posteriormente transportados al paquete de análisis estadísticos SPSS, versión 18.0. Los datos fueron analizados según el objetivo propuesto, considerando estadísticos descriptivos, así como técnicas para estudiar la validez psicométrica de la escala. Seguidamente, se estudió la estructura factorial subyacente a los 14 ítems, con el método de extracción de los Ejes Principales (Principal Axis FactoringPAF). Luego de identificada la solución factorial satisfactoria se procedió a la verificación de la confiabilidad interna, con el estadístico Alfa de Cronbach y la media aritmética de la correlación ítem-total.

\section{Resultados}

A pesar de que el análisis factorial es considerado una técnica robusta (Pasquali, 1999), poco influenciada por fenómenos como falta 
de normalidad de la distribución de las respuestas, se tomó la decisión de investigar la forma en que las respuestas de cada ítem estaban distribuidas. Según Laros (2005) la solución factorial refleja el comportamiento de la medida en la muestra aplicada. Entonces, a mayor asimetría en las respuestas, mayor la probabilidad de encontrar una solución factorial inestable. Por esa razón, la normalidad de la distribución es deseada. Para la verificación de la normalidad de la distribución de las respuestas en los 14 ítems se adoptaron las directrices dadas por Miles y Shevlin (2001), quienes defienden la verificación de la normalidad mediante el cálculo del tamaño de la asimetría (skewness). Los análisis realizados en esta fase mostraron que los datos recogidos tenían distribución bastante próxima de la curva normal. Los valores de asimetría encontrados estuvieron entre -0.74 y -1.5 . De los 14 ítems, solamente 4 presentaron valores superiores a 1, mas no superaron la marca de 1,5 de lo que se concluye que la distribución empírica es bastante similar a la curva normal, y por eso la realización de un análisis factorial es pertinente de ser ejecutado en el conjunto de datos recogidos.

En la segunda etapa del análisis se estudiaron las evidencias factoriales de la matriz de datos, a partir de la matriz de correlaciones, del cálculo del determinante y se verificó el tamaño del índice de adecuación de la muestra $(\mathrm{KMO})$. La matriz de correlaciones mostró presencia de asociaciones significativas entre todos os ítems $(\mathrm{p}<0.05)$ con valores que oscilaron entre 0.27 y 0.66 , evidenciando que las preguntas de la escala estaban relacionadas entre sí, sugiriendo que indagan sobre un mismo constructo subyacente o fenómeno latente. El cálculo del determinante resultó en un valor $<0.0001$ y al ser de escasa magnitud, más diferente de cero, muestra que el conjunto de peguntas posee un elemento común entre ellos (factor). El índice Kaiser-Meyer-Olkin $(\mathrm{KMO})$ se reveló adecuado por mostrar un tamaño de 0.925. Cabe recordar que los valores esperados son próximos de 1 y cuanto más cercanos a ese valor, mejor el comportamiento del conjunto de datos frente a un análisis factorial (Tabachnik \& Fidell, 2007).

Posteriormente, se adoptaron los procedimientos que permitirían reducir las preguntas de la escala a factores con el método de extracción 
de los Ejes Principales (PAF). Se buscó una estructura unifactorial, similar a la de la escala brasileńa, mediante la adopción del criterio de Guttman-Kaiser (Guttman, 1954; Kaiser, 1960) que orienta para que sea observado el valor del eigenvalue del conjunto de datos, el que debe ser igual o mayor que 1.0. Al aplicar este criterio, se observó que era pertinente optar por una solución unifactorial y se adoptó como segundo criterio de definición de solución factorial el test de scree plot, que confirmó la solución unifactorial, dado que había un único punto en que la línea se quebraba. Además de los criterios matemáticos relatados, fue considerada la naturaleza teórica del fenómeno. Al discutir sobre el consenso estratégico relativo a las prácticas pedagógicas, los investigadores de este campo lo asumen como atributo de naturaleza unidimensional. Por tanto, optar por una solución unifactorial se muestra empírica y teóricamente pertinente. Sobre el procedimiento de análisis factorial, Laros y Puente-Palacios (2004) manifiestan que tan importante como la utilización de criterios matemáticos es la verificación de la pertinencia teórica del conjunto de preguntas, pues sin ella no existe justificación alguna para que sea defendida la semejanza de contenido abordado por los ítems que componen la medida. Una vez tomada la decisión de condensar los 14 ítems en un factor único, se prosiguió con la investigación de la contribución que cada uno hace para la comprensión de la percepción de los docentes sobre las prácticas pedagógicas. La extracción de la matriz del factor único mostró que con los 14 ítems se alcanzaba un total de $49.72 \%$ de variancia explicada. La Tabla 2 muestra las cargas factoriales de cada ítem. Esos valores, según Tabachnik y Fidell (2007) deben ser interpretados como la contribución matemática de cada ítem para la escala como un todo. Valores inferiores a 0,30 muestran una contribución pobre o prácticamente inexistente y valores próximos a 1 muestran contribuciones relevantes.

Como puede ser observado, todos los ítems del cuestionario muestran una contribución importante en el proceso de medición del consenso estratégico sobre las prácticas pedagógicas, pues todos ellos superan largamente 0.30 que es considerado el valor mínimo de carga factorial para que una pregunta de una escala o cuestionario sea 
estimado como suficientemente relevante para ser mantenido como parte del conjunto. Según la magnitud de los índices de confiabilidad interna (Alfa y correlación ítem-total), los datos de la Tabla 2 sugieren que las preguntas del cuestionario componen un conjunto único y armónico, de tal forma que los 14 ítems pueden ser reducidos a un solo indicador numérico (media aritmética de las 14 preguntas). Cabe destacar que el comportamiento de las preguntas de un cuestionario puede mostrar contribuciones diferentes en muestras también diferentes. Así, los resultados obtenidos revelan que la presente escala es un instrumento adecuado para mediciones en la muestra de dados recogidos. Habiendo identificado índices de validez satisfactorios se prosiguió con los análisis comparativos. Para realizar esa etapa fueron adoptadas estrategias de investigación sustentadas específicamente en el análisis de variancia. Para Puente-Palacios y Borba (2009) la constatación de la existencia de respuestas semejantes en procesos de diagnósticos grupales es una práctica imprescindible, una vez que arrojará evidencias concretas de que los equipos comparados realmente se muestran diferentes entre sí, y revelara que se ha capturado efectivamente un atributo grupal y no un de naturaleza individual.

\section{Tabla 2}

Cargas factoriales e indices de confiabilidad

Descripción del ítem

Carga factorial

El equipo docente de la escuela...

...trabaja de manera a desarrollar la capacidad crítica de los alumnos.

...trabaja en pro de objetivos comunes.

...actúa de manera compatible con el proyecto pedagógico de

0.72

la escuela.

...incentiva los alumnos a estudiar.

...tiene la calidad de la enseńanza como principal prioridad

0.71 de la escuela.

...incentiva el intercambio de ideas y experiencias entre sus miembros. 
Descripción del ítem

Carga factorial

...se foca en las necesidades específicas de los alumnos de la

0.71 escuela.

...acompaña el aprendizaje de los alumnos.

0.78

...utiliza los resultados de las evaluaciones de desempeño de

0.66

los alumnos para hacer ajustes a las prácticas adoptadas por la escuela.

...utiliza criterios justos para atribuir las notas a los alumnos.

... utiliza recursos diversificados en el proceso de enseńanza -

0.70 aprendizaje.

...explica a los alumnos los métodos de evaluación utilizados en la escuela.

...incentiva la participación de la comunidad escolar en la elaboración de la propuesta pedagógica da escuela.

...es responsable por la elaboración de la propuesta pedagógica de la escuela.

Alfa de Cronbach

Média aritmética de la correlación ítem - total

0.66

El Anova (one way) entre las nueve instituciones educativas mostró la presencia de diferencias significativas entre las escuelas que participaron del estudio $(F=2.52 ; p=.014)$, lo que revela que hay instituciones educativas con mayor concordancia en relación a las prácticas pedagógicas adoptadas y otra con menos concordancia, exhibiendo diferentes niveles de consenso estratégico. No se encontraron diferencias por edad, tiempo de servicio ni sexo $(p>.06)$.

\section{Discusión y conclusiones}

Los resultados observados tornan pertinente defender que la solución unifactorial es la más satisfactoria para la escala de medición del consenso sobre las prácticas pedagógicas. Teóricos del área, al definir el fenómeno, lo describen como atributo grupal de naturaleza global, lo que 
significa que no es ponderada la existencia de diversos núcleos de sentido en la forma de elementos constitutivos del consenso sobre prácticas pedagógicas. Por tanto, a pesar de que las preguntas indagan sobre aspectos diferentes, entre ellas no se identifican claros subconjuntos de asuntos investigados. Los análisis realizados mostraron esa unicidad de los ítems e inclusive la retirada de cualquier pregunta no trae beneficios a la medida.

Los hallazgos revelan una pequeña diferencia en la naturaleza constitutiva de la escala aplicada en los establecimientos educativos en Quito y los datos recogidos en el Brasil. El estudio realizado por Moreira (2011) presentó, también mediante la realización de un análisis factorial exploratoria, que era pertinente retirar un ítem, restando así 13, porque había un que no contribuía adecuadamente para medir la concordancia de los miembros del equipo pedagógico en relación a las prácticas adoptadas por la escuela. Sin embargo, una vez que en un diagnóstico comparativo no hay interés en cotejar cada ítem en separado sino que se compara el conjunto de ítems, estudios de este tipo realizados con datos globales recogidos en Brasil y en Ecuador, son viables y legítimas pues los instrumentos de medida estudiados en los dos países pueden ser considerados equivalentes. Desde una perspectiva teórica, el resultado encontrado se muestra convergente con la naturaleza del propio fenómeno. Al tratarse de un estudio de verificación de las evidencias de validez, se espera que si el proceso de traducción fue correcto, pocas o ninguna diferencia surja, a no ser en el caso de que se indaguen aspectos que son alterados por la idiosincrasia local o, en otras palabras, que sean culturalmente sensibles. Por tanto, la estabilidad o semejanza de la estructura factorial era un resultado esperado, pues se trata de un estudio de adaptación de una medida.

Es necesario destacar los favorables índices de confiabilidad interna encontrados. La magnitud del Alfa de Cronbach (0.92) y de la media aritmética de la correlación ítem-total (0.66) muestran que el cuestionario con sus 14 preguntas constituye una medida útil para el diagnóstico de las actitudes del equipo pedagógico escolar en relación con las prácticas adoptadas. Esta es una información particularmente útil para el gestor escolar en la realización de su tarea de coordinación. 
La relevancia de contar con ese diagnóstico deriva de los resultados de estudios empíricos que muestran asociación positiva entre consenso sobre las prácticas pedagógicas y resultados escolares.

Los resultados de los análisis comparativos realizados muestran convergencia entre los atributos teóricos del consenso. No es suficiente verificar si las actitudes de las personas son favorables en cuanto a las prácticas pedagógicas, sino que es necesario verificar también en qué medida las personas que trabajan en una misma institución educativa poseen visiones favorables compartidas. En este sentido, los resultados mostraron que tanto los docentes, como las escuelas presenta diferencias en relación a las prácticas pedagógicas. La ausencia de diferencias entre los participantes del estudio por atributos se explica dado que las comparaciones se realizaron entre grupos diferentes y no se consideró la demanda de convivencia e interacción exigida para la construcción de cogniciones compartidas.

En términos concretos, las implicaciones prácticas de este estudio son diversas. Por un lado, el gestor escolar puede contar con una herramienta adaptada a la realidad local, que le permite dimensionar en qué medida los miembros de su equipo consideran pertinentes y adecuadas las estrategias adoptadas por la escuela para alcanzar las metas de desarrollo de los estudiantes. Por otro lado, las ventajas de trabajar en un equipo donde los miembros perciben el escenario laboral de manera similar suponen que las cogniciones compartidas ya han sido estudiadas y en esas investigaciones se ha revelado que ellas propician mejor desempeño del equipo (en términos de precisión, eficiencia, calidad del producto) y promueven respuestas afectivas como satisfacción de los miembros. Por lo tanto, se espera que ayuden a mejorar procesos grupales (comunicación, consenso, semejanza de visones, etc.) los que por su vez pueden mejorar el desempeño. Finalmente, su presencia puede ayudar a que surjan resultados en la esfera de la motivación de equipo (cohesión, confianza, eficacia colectiva) que incluso mostrando relación distante con el desempeño pueden servir de elemento de soporte para el bienestar de las personas, el cual es tan importante 
para el éxito escolar como la competencias técnicas (Cannon-Bowers \& Salas, 2001; Lim \& Klein, 2006; McComb, 2008; Rentsch \& Klimoski, 2001).

El conjunto de resultados matemáticos leídos bajo la luz de las teorías del campo de las cogniciones compartidas demuestran que la escala de prácticas pedagógicas es un instrumento con evidencias de validez psicométrica que lo califican para uso en ambientes escolares. Se trata de una herramienta útil para el diagnóstico de las percepciones que el equipo pedagógico tiene sobre las prácticas de trabajo adoptadas en la institución de enseñanza en que labora. Se recomienda continuar los estudios en esta línea con otros grupos humanos, con el fin de analizar la estabilidad de la solución factorial identificada.

\section{Referencias}

Arteaga, M. E. \& Lasio, V. (2009). Empresas dinámicas en Ecuador: factores de éxito y competencias de sus fundadores. Revista Latinoamericana de Administración, 42, 1-14.

Cannon-Bowers, J. A. \& Salas, E. (2001). Reflections on shared cognition. Journal of Organizational Behavior, 22, 195-202.

Cassep-Borges, V., Balbinotti, M. A. A. \& Teodoro, M. L. M. (2010). Tradução e validação de conteúdo: uma proposta para a adaptação de instrumentos. En L. Pasquali y cols., Instrumentação psicológica: fundamentos e práticas. (pp. 506-520). Porto Alegre: Artmed.

Guttman, L. (1954). Some necessary conditions for common factor analysis. Psychometrika, 19, 149-162.

Hackman, J. (1987). The design of work teams. En J. Lorsch (Ed.), Handbook of Organizational Behavior, 315-342. Nueva York: Prentice-Hall.

Kaiser, H.F. (1960). The application of electronic computers to factor analysis. Educational and Psychological Measurement, 20, 141-151. 
Kellermanns, F. W., Eddleston, K. A., Barnett, T. \& Pearson, A. (March 2008). An Exploratory Study of Family Member Characteristics and Involvement: Effects on Entrepreneurial Behavior in the Family Firm, Family Business Review, 21 (1), 1-14.

Laros, J. A. (2005). O uso da análise fatorial: algumas diretrizes para pesquisadores. En: L. Pasquali. (Org.). Análise Fatorial para Pesquisadores (pp. 163-184). Brasilia: LabPAM.

Laros, J. A. \& Puente-Palacios, K. E. (2004). Validação cruzada de uma escala de clima social. Estudos de Psicologia (Natal), 9(1), 113-119.

Lim, B. C. \& Klein, K. J. (2006). Team mental models and team performance: A field study of the effects of team mental model similarity and accuracy. Journal of Organizational Behavior, 27, 403-418.

Mathieu, J. E., Maynard, M. T., Rapp, T. \& Gilson. (2008). Team effectiveness 1997-2007: A review of recent advancements and a glimpse into the future. Journal of Management, 34, 410-476.

McComb, S. A. (2008). Shared mental models and their convergence. En M. P. Letsky, N. W. Warner \& S. M. Fiore, Macrocognition in Teams: Theories and Methodologies (pp. 35-50). Aldershot, UK: Ashgate.

Miles, J. \& Shevlin, M. (2001). Applying regression and correlation: a guide for students and researchers. Londres: Sage Publications.

Moreira, T. F. (2011). O papel do consenso estratégico em equipes pedagógicas. Brasilia: Universidade de Brasilia,.

Pasquali, L. (1999). Testes referentes a construto: teoria e modelo de construção. En L. Pasquali (Org.), Instrumentos psicológicos: manual prático de elaboração. Brasilia: IBAPP.

Puente-Palacios, K.E. \& Borba, A. C. P. (2009). Equipes de trabalho: Fundamentos teóricos e metodológicos da mensuração de seus atributos. Revista Avaliação Psicológica - IBAP, 8 (3), pp. 369-379.

Puente-Palacios, K. E. \& González-Romá V. (2013). Gestão de Equipes de trabalho. En L. Borges y L. Mourão, As pessoas, o trabalho e as organizaçôes: intervençōes a partir da Psicologia. Porto Alegre: Artmed. 
Rentsch, J. R. \& Klimoski, R. (2001). Why do 'great minds' think alike? Antecedents of team member schema agreement. Journal of Organizational Behavior, 22 (2), 107-120.

Swab, R., Postmes, T., Van Beest, I. \& Spears, R. (2007). Perceived shared cognition as a product of, and precursor to, shared identity in negotiations. Personality and Social Psychology Bulletin, 45, 187-199.

Tabachnick, B. \& Fidell, L. (2007). Using Multivariate Statistics. 5ta. edición. Boston: Pearson.

Recibido: 07 de abril, 2013

Aceptado: 28 de mayo, 2013 